\title{
Diversidad Sexual y de Género en Comunidades Educativas de Arica, Chile: Fisura de la Heteronorma desde la Multicultura
}

\section{Sexual and Gender Diversity in Educational Communities of Arica, Chile: Heteronorm Fissure from Multiculture}

\author{
Verónica Rubio-Aguilar * \\ Patricia Miranda Mamani \\ Genoveva Tiayna Pacha \\ Elizabeth Hidalgo Figueroa \\ Carol Tuna Varas \\ Universidad Santo Tomás, Chile
}

\begin{abstract}
La educación es un derecho inherente a todas las personas, que no puede ser coartado por la expresión de una identidad de género diversa al modelo heteronormativo imperante en los currículos escolares, siendo éste un desafío al que se enfrentan las instituciones educativas, requiriendo mayor desarrollo investigativo. Este estudio cualitativo se orientó a comprender los significados que respecto de la diversidad sexual y de género, construyen comunidades educativas de educación básica y media de Arica, Chile. Se utilizó el grupo focal como técnica de recogida de datos, para conocer opiniones de estudiantes; padres, madres y apoderados/as; profesores/as y asistentes de la educación, las que fueron analizadas a través del método comparativo constante de la teoría fundamentada. Los resultados dan cuenta de tensiones en los significados, representadas por la reiteración de una matriz heterosexual heredera de la hegemonía patriarcal en generaciones más adultas que toleran discursivamente las diversidades, pero sancionan su expresión en el ámbito escolar, versus generaciones más jóvenes que las aceptan y reconocen, enfatizando en la urgencia de formación para la transformación social, desde la inclusión y no discriminación de identidades de género que fisuran el binomio femenino/masculino, actuando el multiculturalismo étnico y territorial del contexto investigado como propiciador de estas resignificaciones.
\end{abstract}

Descriptores: Diversidad; Discriminación sexual; Escuela; Multiculturalismo; Educación inclusiva.

Education is an inherent right of all people, which cannot be restricted by the expression of a gender identity different from the heteronormative model prevailing in school curricula, this is a challenge faced by educational institutions, requiring further research development. This qualitative study was aimed at understanding the meanings of sexual and gender diversity in the educational communities of basic and secondary education in Arica, Chile. The focal group was used as a technique of data collection, to know students' opinions; parents and guardians; teachers and education assistants, which were analyzed through the constant comparative method of the grounded theory. The results show tensions in meanings, represented by the reiteration of a heterosexual matrix inherited from the patriarchal hegemony in more adult generations that discursively tolerate diversity, but sanction its expression in the school environment, versus younger generations that accept and recognize it, emphasizing the urgency of training for social transformation, from the inclusion and non-discrimination of gender identities that fissure the feminine/masculine binomial, acting the ethnic and territorial multiculturalism as a propitiator of these resignifications.

Keywords: Diversity; Sex discrimination; School; Multiculturalism; Inclusive education.

*Contacto: vrubioa@santotomas.cl

ISSN: 0718-7378

www.rinace.net/rlei/
Recibido: 28/06/2021

$1^{\text {a }}$ Evaluación: 04/09/2021

Aceptado: $\quad 08 / 10 / 2021$ 


\section{Introducción}

Chile al igual que otros países de Latinoamérica y el mundo se ha adscrito a pactos internacionales que resguardan la integridad de las personas LGBTIQ+ (lesbianas, gays, bisexuales, trans, intersex, queer), tales como los Principios de Yogykarta que buscan garantizar el acceso a la educación en igualdad de condiciones y el trato igualitario de estudiantes, personal y docentes, sin discriminación por motivos de orientación sexual o identidad de género (ICJ, 2007).

Es por ello que desde el Ministerio de Educación, MINEDUC (2017) se reconoce que todas las personas tienen derecho a "ejercer plenamente su sexualidad como fuente de desarrollo personal y decidir autónomamente sobre su vivencia, sin discriminación, violencia o coerción" (p.13), existiendo diversas leyes que intentan asegurar aquello en los contextos educativos, destacando, entre otras: Ley 20.370 general de educación (LGE) (2009); Ley 20.536 de violencia escolar (2011); Ley 20.609 establece medidas contra la discriminación incorporando entre los motivos, la identidad de género y la orientación sexual (2012); Ley 20.845 de inclusión escolar (2015) y Ley 20.911 que establece plan de formación ciudadana para colegios reconocidos por el estado (2016). A las que se suman las Orientaciones para la inclusión de las personas lesbianas, gays, bisexuales, trans e intersex en el sistema educativo chileno (2017) y la Circular $\mathrm{N}^{\circ} 0768$ de 2017 que establece derechos de niñas, niños y estudiantes trans, para asegurar su derecho a la educación y respetar su identidad, a través del uso de lenguaje inclusivo, promoción de espacios de reflexión y capacitación a los integrantes de la comunidad educativa y uso del nombre social, entre otros.

Sin embargo, en la educación formal aún impera un modelo tradicional bajo cuyo alero se construyen planes educativos, que encasillan a las personas desde el binomio femeninomasculino, limitando otras formas de expresión diversas al mandato heteronormativo que como describe el MINEDUC (2017) "es el régimen social y cultural que impone que la heterosexualidad sea la única sexualidad "normal", natural y aceptada y también su correlato: la persecución y marginación de las personas no heterosexuales” (p. 43). Es así como la UNESCO señaló en 2012 que en Chile existiría bullying homofóbico representado en un $68 \%$, correspondiendo a un tipo específico de violencia escolar que se dirige hacia personas por su orientación sexual y/o identidad de género, percibida o real.

En 2016, la Primera Encuesta Nacional de Clima Escolar, efectuada por la Fundación Todo Mejora recoge experiencias de niños, niñas y adolescentes LGBTI, de entre 13 y 20 años, en establecimientos educacionales de Arica a Punta Arenas, donde el 70,3\% de las y los adolescentes manifestó sentirse inseguro/a en la escuela debido a su orientación sexual, el 94,8\% reportó haber escuchado comentarios negativos basados en la orientación sexual e identidad de género entre sus compañeros y el 59,9\% declaró haber escuchado este tipo de comentarios discriminatorios por parte del personal del colegio. En 2017, la encuesta $\mathrm{T}$, que investiga las condiciones de vida de las personas transgénero en Chile, develó que, a partir de una muestra de 315 personas, $50 \%$ de ellas declara haber sufrido violencia y discriminación en el contexto escolar, $56 \%$ señaló que intentó suicidarse y de ellas un $86 \%$ lo intentó entre los 11 a 24 años (OTD, 2017). En términos comparativos, se señala que la probabilidad de ideación e intentos de suicidio es de hasta siete veces mayor entre jóvenes LGBTI que entre sus contrapartes heterosexuales (Martínez et al., 2018). Este dato resulta importante para el presente estudio que se enfocó en comunidades educativas de enseñanza básica y media, donde se ubican niñas, niños y jóvenes hasta los 
18 años aproximadamente. En 2017, en la ciudad de Arica hubo cuatro denuncias por discriminación y 24 denuncias por maltrato en un total nacional de 288 denuncias por discriminación y 2.107 por maltrato (Superintendencia de Educación, 2018).

La investigación anterior efectuada en varios países de Europa y Latinoamérica da cuenta que si bien en algunos casos existe reconocimiento social y jurídico de las personas que expresan diversidad sexo genérica, aún son víctimas discriminación en distintos contextos, siendo especialmente preocupante el acoso que vivencian durante la infancia y adolescencia en espacios educativos, el que tiende a perpetuarse en contextos universitarios y laborales (Ahumada et al., 2018; Alcívar y Cedeño, 2018; Bard Wigdor y Magallanes, 2018; Chávez et al., 2018; García-Orriols y Torrebadella-Flix, 2019; Sánchez González y González-de-Garay, 2020; Vidal Ortiz, 2014).

Algunas investigaciones efectuadas en Chile entre 2014 a 2021 , dan cuenta que persiste la discriminación y la violencia escolar por temas de orientación sexual y de género diversas a la heteronormada, especialmente en el caso de estudiantes trans quienes suelen ser los más marginados dentro del colectivo LGBTIQ+; los y las estudiantes tienden a minimizar los episodios de acoso por orientación sexual, lo cual podría deberse a la naturalización de las conductas discriminatorias al interior de las escuelas; los docentes manifiestan no sentirse preparados para intervenir ante situaciones de discriminación y acoso escolar, evidenciando insuficiencia en sus procesos formativos y desconocimiento en torno a estos temas, así como también vacíos para implementar políticas públicas e institucionales asociadas a la inclusión de la diversidad de género en contextos escolares y de educación superior (Barrientos y Echagüe, 2018; Cornejo, 2014, 2017, 2018; Del Pino Rubio y Verbal Stockmeyer 2015; Espinoza, 2016; Espinoza y Rodríguez, 2020; Galaz et al., 2018; Fundación Todo Mejora Chile, 2017, 2019; Julio et al., 2016; Ramírez y Contreras, 2016; Ravetllat, 2018; Rojas et al., 2019; Rubio et al., 202 1; Salas y Salas, 2016; Toro et al., 2020; Trujillo 2014; Vidal Velis et al., 2020). En síntesis, la investigación sigue siendo escasa y concentrada en la zona central del país, especialmente en la región Metropolitana, con poco desarrollo en regiones extremas como es el caso de la región de Arica y Parinacota.

En este contexto emerge el interés de ahondar en lo que está sucediendo en las comunidades educativas, en tanto, se comprende que la escuela es el lugar donde más tiempo permanece una persona durante la infancia temprana hasta la adolescencia, donde confluyen las primeras experiencias de conformación de identidad de género. Este estudio buscó conocer la pluralidad de opiniones que pueden surgir frente a la diversidad sexual y de género por todos y todas quienes constituyen comunidades educativas, en tanto éstas son agrupaciones de alumnos/as, padres, madres y apoderados, profesionales de la educación, asistentes de la educación, equipos docentes, directivos, entre otros, que se reúnen con el fin de contribuir a la formación y aprendizajes de las y los estudiantes (LGE, 2009).

Interesó específicamente conocer los significados, por cuanto las personas comprenden el mundo mediante la interacción con otros, y con sus entornos cercano y remoto. Los significados traspasan el ámbito de lo privado y se vuelven públicos, dado que las personas participan de su cultura (Bruner, 2006). Esto es lo que ocurre en las escuelas y liceos, puesto que los y las estudiantes, sus padres y madres, ingresan a un contexto donde comparten sus significados, con otros y otras. Por lo tanto, el propósito de este estudio fue comprender cuáles son y cómo se caracterizan los significados sociales que algunas comunidades educativas de educación básica y media de la ciudad de Arica construyen 
respecto de la diversidad sexual y de género, desde la opinión de estudiantes; madres, padres y/o apoderados; profesores, profesoras y asistentes de la educación.

\section{Revisión de la literatura}

\subsection{Diversidad sexual y de género}

La diversidad sexual y de género hace referencia a "todas las posibilidades que tienen las personas de asumir, expresar y vivir su sexualidad, así como de asumir expresiones, orientaciones e identidades sexuales" (CONAPRED, 2016, p. 15). En razón a esto la expresión de género corresponde a "cómo una persona expresa su propio género al mundo, por ejemplo, a través de nombres, ropa, forma de caminar, modo de hablar, de comunicar, de asumir roles sociales y su comportamiento" (UNESCO, 2017, p. 10).

Por su parte, la orientación sexual se entiende como "la capacidad de una persona de sentir una atracción sexual profunda y emocional hacia otros, y tener relaciones sexuales con individuos de un género diferente, el mismo género o más de un género" (UNESCO, 2017, p. 12). En tanto la identidad de género puede ser comprendida como una experiencia individual e interna de una persona sentida íntimamente, que puede o no corresponder con el sexo asignado al nacer (UNESCO, 2016), incluyendo la vivencia personal del cuerpo (que podría involucrar la modificación de la apariencia o la función corporal a través de medios médicos, quirúrgicos o de otra índole, siempre que la misma sea libremente escogida) y otras expresiones de género, incluyendo la vestimenta, el modo de hablar y los modales (Yogyakarta, 2007).

Actualmente cada vez existe mayor reconocimiento de que gran parte de las características que se califican como femeninas o masculinas no son biológicas o naturales, sino adquiridas a través del aprendizaje social e individual (Espinar, 2009). Hoy se comprende que la disconformidad con el género asignado al nacer no es un problema, una enfermedad o un trastorno; trasladando la preocupación hacia el impacto emocional y social que dicha identidad puede involucrar en el curso de vida de una persona, especialmente en contexto de discriminación o exclusión (MINSAL, 2021). Según esto la identidad de género no necesariamente puede ser binaria, sino que puede construirse a partir de la combinación de ambas identidades, la ausencia de ambas, o la incorporación de algún otro género, existiendo variadas formas de expresión y de ser, considerando identidades como, por ejemplo: agénero, bigénero, género fluido, pangénero, además de otras que no se encuentran definidas ni categorizadas (OTD, 2017).

En este sentido, las personas transexuales se sienten y se conciben a sí mismas como pertenecientes al género opuesto que social y culturalmente se asigna a su sexo biológico, sienten rechazo por sus genitales y desean operarse para corregir esta disonancia; los transgénero, quienes a pesar de sentir este desacuerdo entre su sexo físico y psicológico, no sienten la necesidad de modificar sus genitales y los intersex poseen características genéticas y fenotípicas de varón y de mujer, en grado variable (Martínez et al. 2018). También existen personas trans no binaries que no se identifican con los géneros binarios femenino/masculino, independiente del sexo biológico o del género asignado al nacer. En este aspecto Preciado (2002), señala que las personas no conformes con la identidad impuesta socialmente pueden renunciar al género como performatividad y a los beneficios que podrían obtener de dicha naturalización u orden simbólico, existiendo la posibilidad de movilidad de las identidades en múltiples expresiones sexo-genéricas, deconstruyendo 
y tensionando las identidades heterocentradas, desde la eliminación de las categorías biológicas macho/hembra, mujer/varón, en términos legales, sociales y administrativos.

Las conceptualizaciones previas son muy necesarias de analizar, si se considera que la sociedad chilena cisnormada, heterocentrada y patriarcal se ha conformado bajo un paradigma hegemónico que establece relaciones de poder y sumisión entre hombres y mujeres, performando las relaciones entre ambas identidades dejando al margen toda expresión distinta a este tradicional binarismo (Butler, 2002, 2007). Hegemonía que traspasa todos los ámbitos donde se desenvuelven las personas, especialmente el educativo, siendo la escuela el lugar donde se desarrolla gran parte de la socialización primaria y transcurre gran parte de la vida de las personas. Por tanto, se vuelve interesante indagar si la perpetuación de los modelos hegemónicos mantiene inequidades e injusticias a través de relaciones de poder y patrones repetitivos de interacción en el contexto escolar, históricamente reconocido como espacio de disciplinamiento heteronormativo (Cornejo, 2017).

\subsection{Inclusión de diversidades sexo genéricas en contextos escolares}

En la actualidad cada vez es más común conocer casos de niños, niñas y adolescentes que a muy temprana edad, incluso en la etapa preescolar, manifiestan una identidad de género diversa al sexo asignado al nacer y socialmente esperado, provocando un cuestionamiento al interior de las familias y las instituciones educativas. Ante esto se espera que las familias sean el primer espacio de contención y aceptación y que las escuelas se funden en una educación basada en el respeto y valoración de la diversidad y no en un espacio excluyente, inseguro y hostil para quienes expresan una sexualidad distinta a la heterosexual. En este escenario, las instituciones educativas enfrentan la tensión de ser tanto un espacio seguro, al mismo tiempo que son consideradas un espacio de mayor complejidad para quienes asumen identidades no hegemónicas (Ramírez y Contreras, 2016).

$\mathrm{Al}$ respecto la UNESCO (2012) declara que la inclusión de estudiantes que expresan diversidad sexual y de género es un imperativo para evitar la discriminación en contextos educativos, puesto que cada niño, niña y adolescente tiene el derecho de expresar su identidad de género de la forma como él o ella la construya y sienta. Reafirmando la comprensión de la inclusión educativa como "un proceso que responde a la diversidad de las necesidades de todos los alumnos incrementando su participación en el aprendizaje, las culturas y las comunidades, reduciendo la exclusión en y desde la educación” (UNESCO, 2005, p. 13). Por lo tanto, permite que todos se eduquen juntos en igualdad de condiciones, respetando las diferencias y la identidad de cada uno (Blanco, 2006).

\subsection{Significados sociales, constructores de identidades y reconocimiento}

Los significados sociales son formas de interpretar y comprender el mundo y la realidad social incluyendo creencias y valores, que se construyen socialmente en la interacción con otros, situados en un contexto histórico y cultural, que se exteriorizan a través de argumentaciones, discursos y premisas, que connotan las acciones de los individuos (Gergen, 2006). Su formación es influida desde las primeras experiencias que se presentan en la intimidad de la familia, entregando una serie conceptos, juicios y prejuicios que todas las personas de forma inconsciente al principio y de manera consciente después, utilizarán en sus relaciones sociales y que son el resultado y la expresión de la cultura en que nacen y se desenvuelven. Conforme se amplían los contextos de interacción, los significados de la infancia van variando de acuerdo con las nuevas intersubjetividades que se enfrentan al participar del mundo social. 
En este sentido, sentir y expresar una determinada identidad de género en la cultura occidental se encuentra fuertemente influido por significados heteropatriarcales que coexisten con creencias y valores que comienzan a deconstruirlos, dando apertura a una resignificación que respeta y comienza a otorgar reconocimiento jurídico y social (Honneth, 2009) a los derechos humanos y civiles de quienes expresan una identidad de género más allá del binomio femenino/masculino. Pese a la existencia de discursos dominantes que circulan y configuran las relaciones y las prácticas (Revuelta y Hernández, 2019), en que se desenvuelven. Esto demuestra que el conocimiento y la forma de significar el mundo no solo es individual y objetivo, sino que depende de las vicisitudes de los procesos sociales que hacen que prevalezcan unos modelos de comprensión por sobre otros.

\section{Método}

La investigación se sustentó en el paradigma fenomenológico (Vasilachis et al., 2006), utilizando la metodología cualitativa (Salgado, 2007) para favorecer las posibilidades de reflexión (Scribano, 2008) y analizar la particularidad temporal y local del contexto investigado, a partir de las expresiones y actividades de las personas investigadas (Flick, 2012). Para seleccionar a las y los participantes del estudio, se utilizó una muestra intencionada y por conveniencia (Lagares y Puerto, 2001), con el fin de obtener muestras representativas de los grupos específicos que se deseaba investigar, de acuerdo con los criterios de inclusión establecidos, escogiendo: estudiantes de séptimo básico y educación media; profesores/as de educación básica y/o media; profesionales y asistentes de la educación (psicólogos/as, trabajadores/as sociales, asistentes de aula, inspectores de patio) y madres, padres y/o apoderados, conformando con ello un diseño de investigación de casos múltiples (Stake, 2005).

Para resguardar la dignidad, anonimato y confidencialidad, se aplicó a cada estudiante por ser menor de edad, un asentimiento informado y a su apoderado un consentimiento informado para autorizar la participación de su hijo o hija en la investigación. A las y los adultos participantes también se les aplicó un consentimiento informado, previamente aprobado por el Comité de Ética Científico de la Universidad que efectuó este estudio, según certifica carta de aprobación de marzo de 2019.

El proceso de recolección de datos se realizó mediante la técnica de Grupo Focal, con el fin de acceder a la dimensión práctica de los mundos sociales (Canales, 2006), explorando los significados a partir de la interacción entre las y los participantes, agrupando sus conocimientos, prácticas y opiniones, para saber cómo piensan y por qué piensan lo que piensan (Flores, 2009). Se utilizó el mismo guion temático para todos los grupos focales, el que antes de su uso definitivo fue validado por una experta en metodología cualitativa y probado mediante prueba piloto con sujetos con similares características a las muestras seleccionadas. Los contenidos del guion temático se organizaron de acuerdo con los objetivos del estudio, orientados a conocer el contexto socio histórico cultural; la dinámica de relaciones interpersonales y la construcción de conocimientos en torno a la diversidad sexual y de género, que ocurren al interior de las comunidades educativas.

El trabajo de campo se situó en cuatro comunidades educativas de la ciudad de Arica, de carácter municipalizado de orientación laica, seleccionando dos establecimientos de enseñanza básica y dos establecimientos de enseñanza media (un liceo científico humanista y un liceo técnico profesional). Las escuelas tienen un índice de vulnerabilidad escolar en 
promedio de $85 \%$ y los liceos de $92 \%$. Escuelas y liceos cuentan con equipos de integración escolar (PIE) y convivencia escolar. En su conformación estudiantil, son establecimientos mixtos, hay estudiantes extranjeros, principalmente peruanos y bolivianos, $\mathrm{y}$ pertenecientes a pueblos originarios y afrodescendientes. En las escuelas participaron estudiantes de séptimo básico, de 12 a 14 años y en los liceos, estudiantes de primero a cuarto medio, de 15 a 18 años. Con relación a madres, padres y/o apoderados, todos poseen educación básica y media completa, pertenecen a un rango etario entre los 35 a 60 años, son trabajadores dependientes, otros independientes o desarrollan trabajos informales. Por su parte, los profesores/as de educación básica y/o media; profesionales y asistentes de la educación, poseen formación universitaria completa y se ubican en el rango etario entre los 40 a 60 años.

Entre los meses de marzo a mayo de 2019, se llevaron a cabo seis grupos focales, integrados por participantes de las cuatro comunidades educativas, organizados por estamentos (madres, padres y/o apoderados; profesionales y asistentes de la educación; estudiantes) y tipos de establecimientos (básica o media), con el fin de favorecer la confianza entre personas con similares características. Cada grupo focal estuvo conformado por 10 a 12 personas y tuvo una duración aproximada de una hora y treinta minutos.

Los datos recogidos, fueron analizados con el método comparativo constante de la teoría fundamentada (Strauss y Corbin, 2002), lo que permitió aproximarse inductivamente al fenómeno de interés, desarrollando un análisis descriptivo, relacional e interpretativo, mediante el uso de las codificaciones abierta, axial y selectiva, lo que permitió formular una explicación de la realidad investigada y de la variación entre categorías. Los análisis elaborados fueron validados a través de un proceso de triangulación múltiple: intersubjetiva entre las investigadoras; con las personas investigadas y con una profesional experta en metodología cualitativa.

\section{Resultados}

\subsection{Legado patriarcal versus generación millennial}

Esta categoría alude al contexto socio histórico cultural en que ocurre la construcción de significados frente a la diversidad sexual y de género que elaboran las comunidades investigadas. Se caracteriza por la presencia de dos polos de un mismo continuo que enfatiza valores y creencias de una sociedad patriarcal presente en las personas más adultas versus cuestionamientos a las desigualdades e inequidades de género en quienes representan a la generación calificada como millennial por las mismas personas investigadas. Por tanto, el análisis de los relatos se tiñe desde una perspectiva generacional.

Identifican particularidades del macro contexto que inciden en sus significados, el primero es el patriarcado, en que predomina la perspectiva de poder autoasignado del hombre por sobre la mujer, predeterminando roles y posiciones de privilegio y subordinación en los ámbitos público y privado respectivamente, reproduciendo desigualdad de género.

También creo que, en el matrimonio, es como machismo, de que los hombres trabajan $y$ las mujeres cuidan a las guaguas. To digo que tiene que haber igualdad. (Estudiante 5) 
¿Quién no está orgulloso de su mamá? Y sin embargo nuestra mamá ¿Qué es lo que era? Mi mamá, dueña de casa excelente, cocinera, mamá y muchas cosas más dentro del hogar. (Apoderado 1)

Un segundo elemento que califican como represivo y opera como dispositivo de control heterosexista, es la religión, que desde los distintos credos que se profesan mayoritariamente en Chile, les ha inculcado que todo aquello que se aleja de la heteronorma, es considerado un pecado, debiendo solo valorar la unión entre un hombre y una mujer, especialmente dentro del vínculo del matrimonio. De esta forma, sería impensado y sancionado aprobar otras formas de relaciones, lo que denota una concepción esencialista del género y una valoración de las expresiones socio afectivas reducidas a las relaciones heterosexuales.

Que Dios creó al hombre y la mujer para que se reprodujeran y no que estuvieran con hombres o con mujer. $Y$ creo que eso hace sentir mal a los bisexuales, homosexuales, los hacen sentir apenados y discriminados. (Estudiante 6)

Los participantes más jóvenes expresan que existiría rechazo en las personas más adultas de las comunidades educativas respecto de reconocer la diversidad sexual y de género como una forma válida de expresión de identidad. Esto ocurriría porque durante décadas la diversidad ha sido invisibilizada por la matriz heterosexual binaria dominante en las familias chilenas, tradicionales y conservadoras.

En mi casa mi abuela, casi nadie de mi familia que sea adulto no les gusta no, no están de acuerdo con eso, lo mismo que dijo ella, mi mamá también lo dice "es que son muy chicos", "no pueden decir eso", o de que no le puede gustar su mismo género, cosas así. Ellos como que están en contra y no, eso. (Estudiante 5)

Los y las estudiantes en su totalidad, algunos profesores/as, madres, padres y apoderados, comprenden la expresión de la diversidad sexual y de género como algo íntimo y personal, que no necesariamente debe regirse por estereotipos sociales binarios, demostrando una opinión crítica al respecto. Esto se ampara en el establecimiento de convenios y tratados internacionales a los que se ha adscrito Chile en la última década y que sostienen el marco legal reciente instaurado en el país, que comienza a resguardar la integridad de las personas y su derecho a la libre expresión de la identidad sentida.

Se está viendo que hay un avance por lo menos, que el Gobierno lo está viendo, lo está considerando por lo menos con las leyes. Porque qué pasa antes, las personas que eran homosexuales se reprimían, no sé, por toda su vida o quizá, tenían una doble vida, pero no lo hacían público, por miedo a qué, a la sanción social, por miedo a muchos factores, que por eso mismo se escondieron. (Asistente de la educación 3)

Refieren que este reconocimiento, aunque incipiente aun, se observa al interior de lo que denominan familias modernas percibidas como libres de prejuicios, con mayor acceso a información y una dinámica familiar que promueve el diálogo, la escucha activa, espacios de reflexión y comunicación entre padres e hijos, lo que favorecería una co-construcción horizontal sobre la diversidad, logrando desapegarse de la herencia patriarcal.

To vengo a hablar un tema personal. Mi hijo se considera bisexual ¿̇ya? Está en la básica, séptimo año ¿y cómo lo supe yo? le pregunté “¿te gustan los niños?”, "si, mamá”, "pero antes te gustaban las niñas", "es que me gustan los niños y las niñas”, "¿tú te consideras bisexual?", "si, mamá" me dice. To le digo que tiene que tener la confianza para decirlo, es un sentimiento natural, que nace desde adentro tuyo, no hay nada malo en eso. $\Upsilon$ de a poco así, fuimos conversando hartas cosas, porque yo tampoco puedo imponerle cosas a él, si es su sentir, es algo natural, de él. (Apoderada 6) 
Expresan que esta tendencia a la inclusión de la diversidad es validada por lo que denominan generación millennial, refiriéndose a quienes nacieron entre 1981 y 2000. Explican que son personas que valoran y respetan toda forma de vida, como diferentes expresiones de género y socio afectivas, son los llamados ambientalistas y animalistas de la generación dos mil. En lo referido a la diversidad en su amplia dimensión y principalmente a la sexual y de género, no demuestran una actitud cuestionadora ni cargada de prejuicios porque no los tienen, sin embargo, sí poseen amplia conciencia de los cuestionamientos de generaciones mayores hacia estas manifestaciones.

Los adolescentes, que son millennial, vienen con un chip, vienen con una apertura natural. Pero los que vienen antes, no lo traen ¿̇Por qué? Porque la influencia parental es tan fuerte, porque ha sido tan fuerte desde atrás. (Asistente de la Educación 10)

Hay que hacerle entender a los adultos de cómo somos. (Estudiante 9)

Los niños son tolerantes, son mucho más tolerantes. Están abierto a nuevas experiencias. $Y$ es algo de lo que no se hacen problemas, somos nosotros, los adultos los que nos hacemos el problema, somos los adultos los que vemos cosas, los niños no ven nada. (Apoderado 7)

Debido a esta divergencia de perspectivas, derivadas de mandatos tradicionales versus posmodernos, se producirían fricciones en las familias y en las comunidades educativas. Esto pone en conflicto a las personas que manifiestan identidades fuera de la heterocisnorma, respecto de la decisión de expresarse libremente o autocensurarse, en una cultura que, desde distintos espacios y agentes, al mismo tiempo, les acepta, rechaza, protege y sanciona.

Hay un claro conflicto, no lo podemos negar. Hay un problema, hay un drama detrás,
cuando tú te sientes obligado a, por algo que no es tu naturaleza, desde tu formación
familiar, de viejos como nosotros, abuelitos, que tenemos otra trascendencia tachada,
el tema aceptar pasa por un poco obligar, que no lo trasmitimos, por lo tanto, no lo
traemos. Y ese conflicto es real, si no, no tendríamos que tener leyes para respetar esta
diferencia. (Asistente de la Educación 9)

En complemento, expresan que quienes dirigen las escuelas investigadas y toman decisiones, lo hacen desde el mantenimiento de patrones heterosexistas puesto que etariamente serían herederos del patriarcado. Por tanto, no existiría aún un proyecto educativo en las escuelas investigadas que incorpore como eje estratégico de formación, la inclusión sustantiva de la diversidad sexo genérica, retrasando los potenciales avances en este ámbito en el contexto local, pese a la existencia de normativas y orientaciones ministeriales para propiciarla.

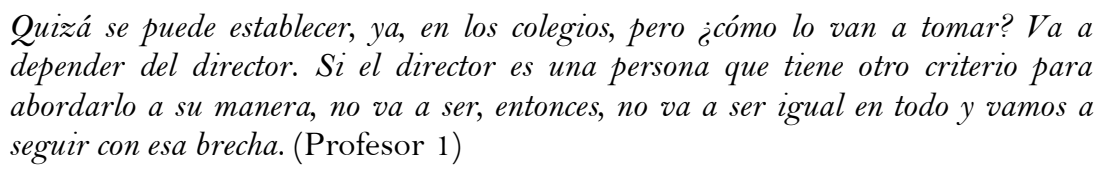

\subsection{Interacciones en la escuela: entre el discurso oficial y el currículum oculto}

En esta categoría se alude a la dinámica de relaciones interpersonales entre los diferentes estamentos de las comunidades investigadas, respecto de la inclusión de personas que externalizan diversidad sexual y de género, la que acontece entre discursos oficiales que promueven la inclusión versus prácticas pedagógicas ocultas que la frenan. Entonces si bien se aboga por una educación basada en el respeto irrestricto que valora las diferencias, prevalecen prejuicios que regulan las interacciones sociales, desde la heteronorma que valida la supremacía masculina por sobre la subordinación femenina, siendo el mandato de acatamiento mucho más enérgico cuando se trata de personas que expresan una identidad 
de género diversa a la atribuida al nacer y socialmente esperada. La reproducción ritualizada del sistema heterocisnormado como régimen social y regulatorio establece roles y dinámicas de interacción que estereotipan lo que deben cumplir hombres y mujeres, lo que se perpetúa en la escuela, tal como lo expresaron las y los representantes de las comunidades investigadas, en cómo debe vestirse el uniforme escolar más restringido para la mujer y más libre para los hombres y en la dictación de clases de educación física desarrolladas por profesoras para alumnas y profesores para alumnos, entre otros ejemplos. Por tanto, se aprecia poco espacio para expresiones fuera de estos binarismos regulando las interacciones sociales solo entre hombres y mujeres y connotando lo femenino como una categoría inferior a la masculina.

To por ejemplo me muevo por muchas salas, entonces voy observando, uno se da cuenta que pareciera ser un castigo para los niños, los de prebásica, que cuando se portan mal, los pongan en la fila de las mujeres, porque están todos alineados, todos los hombres acá, todas las mujeres acá. Entonces, el abordaje, ahí es cuando eres niño, niña, y los separamos y si te portas mal te pongo en la fila de al lado, para que entienda que es un castigo. (Asistente de la educación 4 )

Las estrategias para regular la dinámica de las relaciones interpersonales simulan ser inclusivas desde sus declaraciones, pero siguen perpetuando el binomio masculino/femenino desde las prácticas sociales y pedagógicas que ocurren en la trastienda de dichas argumentaciones. Por lo tanto, las interacciones entre los distintos estamentos suelen estar interferidas por discursos inclusivos aparentes, más no desde un genuino respeto y reconocimiento a las diversidades, profundizando aún más las desigualdades de género.

Como en educación física, nos separan hombres y a mujeres. $Y$ cuando nos separan, le pregunto a la profesora, “¿Por qué no pueden hacerlo hombres y mujeres?” y me dice que no, mujer y hombre separados. (Estudiante 5)

La dinámica de las relaciones interpersonales ocurre desde perspectivas generacionales antagónicas que la regulan formal e informalmente. Así las generaciones más adultas le restan espacio a la existencia de un discurso que promueva oficialmente interacciones inclusivas, mientras que las generaciones de estudiantes demuestran mayor tolerancia a las diferentes expresiones de identidades de género.

En la sociedad está el problema, por un tema generacional, porque los niños no se hacen mayores problemas, que vean algo fuera de lo común, si los adultos, que quizá son, por los tiempos que por la generación que es distinta, pero un problema transversal es la comunicación. (Asistente de la educación 3)

Una característica que favorece la apertura de interacciones que promueven la inclusión, especialmente entre estudiantes, es la existencia de multiculturalismo en el territorio donde se asientan las comunidades investigadas, puesto que al ser la región de Arica y Parinacota una zona fronteriza que limita con otros países se transforma en un corredor natural de migrantes de Perú, Bolivia, Venezuela y Colombia, entre otros. A esto se une la convivencia permanente e histórica con estudiantes de pueblos originarios y afrodescendientes, lo que sin duda colabora en comprender a las diversidades sexo genéricas, como otra arista más que puede atribuirse a la concepción de diversidad.

Las identidades indígenas, eh las identidades no sé migrante, eh las identidades urbanas también etc. entonces también viene a sumarse esto, esta nueva como categoría o nomenclatura que existe que son los LGBT etc... cada vez que converso con un estudiante y le digo "esto es lo que vamos a trabajar en el año, la unidad que más le interesa es la de la diversidad sexual y quieren participar en ella y todos... y profesor 
y cuando vamos a llegar a ese tema, y yo he tenido que actualizarme, ya en base a ello, para poder cumplir sus expectativas. (Asistente de la Educación 2)

Se le da esta importancia a la multiculturalidad también nosotros igual celebramos hartas actividades ya cada niño si quiere expresar algo quieres ser algún número alguna cosa se deja se le da su espacio y no se trata mal. (Estudiante $\mathrm{N}^{\circ} 9$ )

Uno de los puntos esenciales de formación ciudadana es identidad y cuando hablamos de identidad cultural bueno todo lo que forma la persona y de ahí se habla mucho del tema quizás tabú o que no se sale mucho a la luz. (Estudiante $\mathrm{N}^{\circ} 5$ )

Hay una gran aceptación a los cambios que hemos querido hacer los aniversarios anteriores o sea a estos aniversarios comunes que ahi nosotros somos uno de los aniversarios más artísticos más innovadores y también inclusivos...si ñusta y tocayo entonces hemos querido sacar de esos límites al Liceo el tiempo de aniversario por lo menos ese es como nuestro granito de arena que podemos dar a los niños y que por ahí se parte yo creo desde poquito nosotros mismos. (Estudiante $\mathrm{N}^{\circ} 13$ )

\title{
4.3. El reto de la formación para la inclusión de las diversidades
}

En esta categoría se reúnen los conocimientos que frente a la diversidad sexual y de género detentan las comunidades investigadas. En primer lugar, se aprecia una nueva forma de entender la sexualidad humana más allá del reduccionismo biologicista reproductivo heredado y en segunda instancia, se advierte la urgencia de actualización de conocimientos en los y las profesionales de la educación, padres y madres, como imperativo para una adecuada comprensión de las diversidades de género que permita su efectiva inclusión en las instituciones educativas y en la familia. En Chile existen orientaciones emanadas desde el Ministerio de Educación para la inclusión de personas LGBTIQ+ en todos los niveles educativos, por tanto, los establecimientos deben velar por aquello. Sin embargo, en los relatos se devela que sólo dos de las cuatro comunidades investigadas aplican estrategias, que trascienden lo informativo y se traducen en acciones hacia la población LGBTIQ+, incorporando a madres, padres y apoderados. No obstante, estas actividades no son permanentes ni son parte del proyecto educativo, son más bien acciones aisladas que responden a un incipiente acercamiento, desde el abordaje solo en algunas áreas e incluso implementadas por agentes externos a las comunidades, como lo son estudiantes en práctica de disciplinas de las ciencias sociales.

\begin{abstract}
Anualmente se trabaja en algunas de las áreas, yo creo que, en orientación, donde de manera colaborativa con redes se hacen talleres de diversidad sexual o de sexualidad a los estudiantes. El año pasado tuvimos la experiencia de que fue dirigido para padres y apoderados y madres, también para los asistentes de la educación. $\Upsilon$ eventualmente las chicas que estaban en práctica de trabajo social hicieron los talleres para los estudiantes. (Asistente de la educación 3)

Acá tenemos a chicos trans y pese a que no hay baños mixtos, sí se permite que los chicos y las chicas que hacen la transición puedan utilizar los baños correctamente a su género social, a su identidad de género. (Asistente de la educación 6)
\end{abstract}

Existe en los profesores/as y profesionales de la educación, escaso conocimiento de las orientaciones y normativas emanadas por el Ministerio de Educación, lo que no colabora en proteger los derechos de estudiantes trans, como tampoco en propiciar espacios formalizados para su inclusión en los contextos investigados. Esto podría estar reflejando un ámbito de gestión no desarrollado en estas comunidades educativas locales.

Yo no sé si en el reglamento de la escuela, lo que está en los manuales, sale algo de la inclusión a los niños con diversidad sexual żhay algo que habla de forma explícita sobre el tema? (Profesor 1) 
Las madres, padres y profesionales de la educación señalan la necesidad de capacitación en diversidad sexual y de género, ya que los estudiantes demostrarían ventajas comparativas al detentar mayores conocimientos que las y los adultos. Lo que demuestra la urgencia de adquirir información que les permita poseer un bagaje apropiado a nivel conceptual y a nivel estratégico-interventivo, frente a expresiones de diversidad en la comunidad educativa.

Como dice la colega, que nos preparen a nosotros con herramientas, con competencias para poder enfrentar, poder nosotros mismos enseñar porque si empezamos desde los niños y nosotros no tenemos las competencias ¿̇qué pasa? No vamos a ser capaces de enseñar o de explicar realmente como son las cosas. (Asistente de la Educación 7)

La falta de formación que expresan poseer las y los profesionales de la educación, va en desmedro de otorgar una adecuada educación a la comunidad educativa, lo que se refleja en una confusión y reduccionismo de conceptos. Refieren que existen una serie de precisiones conceptuales que no identifican ni manejan adecuadamente. Ante esto buscan resolver su necesidad de formación en redes sociales no formales, donde se privilegia la inmediatez de la información, pero que no ayuda cuando se requiere otorgar una adecuada atención a estudiantes que vivencian sus tránsitos identitarios en los contextos escolares.

Tenemos una serie de profesionales que lo abordan de manera distinta, uno con mejores habilidades, otros que no, te están hablando de lo que es la identidad, de lo que es ser transexual, lo que es el travesti, para hacer una diferenciación, para que los niños entiendan, porque muchas veces como dice el colega, hay profesionales que no tienen los conocimientos o las competencias para poder abordar esos temas y hay confusiones, hay errores. (Asistente de la educación 6)

Esta falta de formación según relatan sería más preocupante en el caso de las y los integrantes más adultos de las comunidades educativas, quienes han internalizado significados de sexualidad que hoy serían obsoletos, tales como la concepción meramente biologicista y reproductiva de los géneros. Les inquieta que estas personas en la mayoría de las comunidades investigadas son quienes ocupan cargos directivos y poseen significaciones que declaran son difíciles de deconstruir, lo que afectaría las posibilidades de generar espacios efectivamente inclusivos, en lo que refiere a identidades diversas e incluso en otros ámbitos de inclusión.

Ese es el problema, ya tenemos criterios de conceptos ya definidos, que es difícil como modificarlos. Como antes cuando les decían a las personas con capacidades diferentes, no sé, discapacitado o el síndrome de down, mongolito. Ahora estamos categorizando otros conceptos, pero yo creo que, con el tiempo, ya va a ser después, ya no se va a hablar de heterosexualidad o de transexualidad, o sea, va a ser algo, un tema que se va a pasar no más, que vamos a conversar como estamos acá. (Asistente de la educación 1)

\section{Discusión}

La gran categoría central que emerge del análisis refiere tensiones en los significados que construyen algunas comunidades educativas de Arica, Chile, respecto de la diversidad sexual y de género, dando lugar por una parte a la mantención de la hegemonía heteronormada y, por otra parte, a la fisura de esta (Butler, 2007), lo que comporta que exista una inclusión aparente en los discursos, pero no una inclusión sustantiva en las prácticas sociales y educativas, de acuerdo a lo expresado por las personas investigadas. Es decir, existiría inclusión en el discurso, pero no en el patio de la escuela, según puede interpretarse desde los relatos analizados. Estas dos visiones contrapuestas, responden a 
un contexto socio histórico actualmente en tránsito, en que confluyen tanto, perspectivas tradicionales que constriñen solo a dos las identidades de género, como miradas postmodernas que amplían el espectro de las expresiones identitarias más allá del dualismo femenino/masculino.

Por tanto, dependiendo de características etarias, generacionales, de ideología religiosa, de roles y posiciones, las personas demuestran diferentes significados que las sitúan en distintos lugares de las tensiones descritas. Se estaría entonces en un escenario de deconstrucción y resignificación que otorga reconocimiento (Honneth et al., 2016) a las diversidades sexo genéricas, al mismo tiempo que desmonta y desnaturaliza mandatos heteropatriarcales que insisten en mantenerse imbricados en los contextos escolares. Al respecto Rojas y otros (2019), refieren que, dependiendo de las posiciones de clase social, género, territorio, formación académica, experiencia política, creencias religiosas y edad, los sujetos demuestran, experiencias más o menos cercanas con la temática y niveles de reflexividad muy distintos.

Las tensiones identificadas no son problematizadas en profundidad por quienes conforman las comunidades investigadas, más bien existiría un acuerdo tácito, donde ningún integrante invade los significados del otro, intentando convivir en una pseudo-armonía. Las y los profesores y asistentes de la educación de generaciones contemporáneas son explícitos en declarar que los discursos oficiales de las comunidades educativas reproducen binarismos de género en las prácticas sociales y pedagógicas, agregando que la decisión final de cómo actuar frente a las diversidades, la tomará siempre quien dirige la escuela y frente a eso sólo queda acatar, por temor a perjuicios laborales.

El segmento más adulto mantiene la concepción binaria como única expresión válida, cuya tarea es la regulación y autorregulación de la conducta en relación con la identidad de género socialmente asignada y esperada. Por tanto, estiman que escapar a la heteronorma provocaría un desequilibrio en los espacios familiares y escolares. Detentan significados de invisibilización, rechazo y segregación frente a las diversidades sexuales y de género, siendo difícil aceptar el cambio paradigmático que actualmente les otorga reconocimiento, puesto que su crianza, socialización y gran parte de su vida ha transcurrido bajo el mandato patriarcal. De este modo, se perpetúa la violencia homofóbica que impera en la escuela (Dinis, 2011) y ésta sigue actuando a decir de Cornejo (2017) como agente de disciplinamiento heteronormado, donde el silencio sobre la diversidad sexual es el reverso de la naturalización de la heteronormatividad, impidiendo o limitando los espacios para que otros sentidos de las sexualidades puedan expresarse. Similares resultados se aprecian en los estudios de Trujillo (2013) en que se identifica resistencia frente al tema de la diversidad sexual y prejuicios de parte de la planta docente, mientras que Rojas, et al. (2019), declaran que la hegemonía de un discurso heteronormativo está presente fundamentalmente en la generación adulta.

En contraparte, quienes expresaron significados de reconocimiento de las diversidades sexo genéricas, son representantes de una nueva generación, conformada por estudiantes, profesores, madres, padres y asistentes de la educación, nacidos en una época en que el modelo educativo heteronormado es cuestionado como una única opción. Son los llamados millennial, nacidos entre los años 1981 y 2000, aproximadamente, que no solo se distinguen en términos cronológicos, sino que son una generación que dista sociológicamente de las anteriores, por su apertura a un nuevo mundo, más amplio y diverso, por ser los primeros en tener acceso a internet y a un mundo globalizado. (Laviosa 
2016). Si bien se educan y educaron en contextos regulados por el binarismo de género, durante su crecimiento y conformación de identidad han estado expuestos a transformaciones sociales, políticas y culturales, que deconstruyen el pensamiento dicotómico tradicional. Estas transformaciones han tenido su origen en algunos episodios de connotación pública de discriminación y violencia a las diversidades y disidencias, tales como la muerte de Daniel Zamudio en 2011, que propició avances legislativos como la ley 20.609 de 2012 que establece medidas contra la discriminación. En complemento las luchas de los movimientos feministas y LGBTIQ+ les han permitido comprender los derechos de las diversidades como legítimos y plausibles, desterrando concepciones binarias, que van perdiendo validez empírica y otorgan espacio a resignificaciones. No vivencian el peso de la cultura patriarcal con la misma opresión que sus ancestros, incorporan y naturalizan la diferencia de unos con otros, cuestionan y rechazan la homogeneidad, junto con detentar mayor manejo de conocimientos sobre identidades de género. Poseen significados de apertura a la diversidad, a diferencia de los adultos, a quienes incomoda y consideran que la expresión de identidades diversas es algo íntimo y privado de las personas y no un tema que haya que formar y visibilizar en la escuela.

La inclusión de personas que expresan diversidad sexual y de género en las escuelas y liceos investigados no se asienta total y formalmente desde las directrices de la política pública y marco regulatorio vigentes, sino que se nutre desde los conocimientos de los y las estudiantes y nuevas generaciones de profesores y profesoras, al mismo tiempo que se fragiliza por la ausencia de actualización de saberes en las y los profesionales de la educación y generaciones más adultas. En términos pedagógicos y de intervención psicosocial el tema se aborda de manera incipiente, a través de acciones aisladas y esfuerzos individuales. Por tanto, son más bien prácticas de integración que de inclusión. Esto puede ocurrir porque las políticas hasta ahora emanadas desde el MINEDUC han tendido a la integración, incentivando una aceptación pasiva sin reconocimiento explícito de la diversidad sexual y de género (Cornejo, 2018).

Esto resulta preocupante si se compara con algunas investigaciones, tales como la del MOVILH (2010), Trujillo (2013), Julio y otros (2016) y Toro y otros (2020), entre otras, en que las y los profesores señalaron no recibir ningún tipo de capacitación, no estar preparados para prevenir y responder efectivamente, ni disponer en sus centros de estudios, de políticas que aborden directamente la inclusión de la diversidad en contextos educativos, lo que demuestra que en prácticamente una década no existen cambios significativos en Chile respecto de la inclusión de las diversidades sexo genéricas en los contextos escolares. Por tanto, no se demuestra aún la existencia de políticas institucionales que propicien la inclusión, siendo perentorio su implementación para colaborar en la transformación social a que urge la sociedad actual. Lamentablemente las políticas educativas actuales aún ofrecen orientaciones muy generales respecto de las identidades de género y las orientaciones sexuales diversas, desde un discurso muy abstracto que muestra avances en aspectos sociales, sin impactar las lógicas de enseñanza (Rojas et al., 2019).

A decir de Junqueiras ,en Barrientos y Echagüe (2018), los dispositivos legales que regulan la violencia en las instituciones educativas no logran problematizar el carácter estructural de las diferencias sociales reproducidas en la escuela que concluyen en tratos violentos, arbitrarios y de exclusión, lo que produce un tipo de pedagogía orientada a con-vivir con lo diferente, reproduciendo el mutilado ejercicio de ciudadanía de las personas noheterosexuales. Ahí es donde gana terreno el currículum oculto que invisibiliza las 
diversidades de género en el aula y en el patio de las escuelas y liceos, manteniendo prácticas de exclusión que cohabitan con microdiscursos que simulan inclusión, pero que finalmente reproducen los macrodiscursos generalistas e imprecisos de las actuales políticas educativas, dejando poco espacio para la deconstrucción de significados que fisuren la heteronorma y consoliden una educación efectivamente inclusiva. Pese a la adscripción de Chile a pactos internacionales que progresivamente otorgan reconocimiento afectivo, jurídico y social a las diversidades sexo genéricas, paradójicamente se convive con prácticas que continúan reproduciendo desigualdad en los contextos escolares.

En este sentido, desde la perspectiva interseccional (Crenshaw, 1989), es posible analizar la reproducción institucional de la desigualdad (Expósito, 2012; Gil 2011; Hill Collins, 2017) que ocurre en las comunidades educativas, situando a quienes expresan diversidad sexo genérica en posiciones de desventaja y discriminaciones múltiples (Romero y Montenegro, 2018). En el caso de este estudio desde la intersección de las categorías de género y edad. Respecto de la primera cuando se rechazan las identidades no heterosexuales y desde la segunda cuando desde la perspectiva adulto-céntrica de quienes dirigen las escuelas y liceos, se regula y constriñe la expresión sexo genérica de sus estudiantes dentro del binomio femenino masculino. De este modo, fue posible vincular las bases de la discriminación desde la experiencia situada (Rivera, 2016) de las comunidades investigadas, al indagar y examinar los significados que construyeron las y los distintos actores/as que las conforman. Esto permitió poner el acento sobre la tendencia que tienen los significados y discursos a adoptar posiciones hegemónicas que comportan exclusiones y cosas no dichas o simuladas (Viveros, 2016), en este caso respecto de las identidades que fisuran la heteronorma.

Según Hill Collins y Hilge (2016) las dimensiones estructurales (instituciones y leyes, en este caso escuelas y políticas educativas), disciplinarias (administración y gestión pública, por ejemplo, normas de convivencia), culturales (hegemonías e ideologías, por ejemplo, heteropatriarcado) e interpersonales (interacciones entre individuos, en este caso de quienes conforman las comunidades educativas) operan tanto de manera singular como en combinación, en la configuración de la organización social del poder y con ello en la expresión de sistemas que desde la regulación institucional, cultural y política articulan ciertas prácticas de discriminación y exclusión de las diversidades. De esta forma las desigualdades sociales toman la forma de opresiones que se cruzan organizadas a través de dominios de poder, configurándose las comunidades en el contexto en el que las personas experimentan estas relaciones de poder y le dan sentido a las experiencias individuales y colectivas que ocurren dentro de los arreglos jerárquicos de poder (Hill Collins, 2017). Como pudo advertirse en las comunidades investigadas en que, a través de las interacciones entre sus distintos actores, al mismo tiempo se cruzan, se reproducen y se combaten relaciones de poder en torno a las diversidades sexo genéricas.

\section{Hallazgos y conclusiones}

Un acierto de este estudio fue la consideración de la opinión de todas y todos quienes conforman las comunidades educativas, aun cuando los datos no son generalizables, es una aproximación fiable a los significados de diversidad sexual y de género que construyen distintas y distintos actores educativos, lo que permite identificar claroscuros en cada uno de los grupos estudiados, para desde allí contribuir a una educación verdaderamente 
inclusiva. En tanto son las comunidades educativas entornos privilegiados donde se vivencia y se tornan posibles proyectos inclusivos (Cornejo, 2018).

Cabe destacar el hallazgo de multiculturalismo que caracteriza la zona investigada, norte de Chile, puesto que si bien esta investigación presenta algunos resultados similares a los de estudios previos en el país, se diferencia y da cuenta en forma particular de cómo este elemento colabora a una resignificación que otorga reconocimiento a las diversidades sexo genéricas, específicamente desde las generaciones más jóvenes, al incorporarlas junto con la etnicidad y la migración en sus vidas cotidianas, al habitar un territorio que se caracteriza por la convivencia histórica de distintas culturas.

Las comunidades investigadas ubicadas en la zona fronteriza, región de Arica y Parinacota dan lugar a una cultura que incorpora las diferencias en sus proyectos educativos, otorgando un sello distintivo y propio de este territorio, que mantiene relaciones permanentes con peruanos, bolivianos y afrodescendientes, respeta y preserva las culturas originarias en lugar de invisibilizarlas e intentar homogeneizarlas mediante estrategias de asimilación. Lo que sin duda marca una distinción respecto de la zona central del país notoriamente más permeable a culturas foráneas que a originarias, predominando hegemonías como el heterocentrismo que justifican las desigualdades. Parafraseando a Hill Collins (2017) se podría decir que las comunidades investigadas comparten una historia y una cultura dentro de un sistema mayor de dominación, frente al que establecen ciertas estrategias de resistencia ante dicho ejercicio de poder, en este caso de la colonización heteropatriarcal.

Parte de esta apertura a las diversidades en el contexto investigado puede explicarse por la influencia de la cultura aymara que comienza a migrar en la década de 1960 desde la cordillera de los Andes hacia las ciudades costeras (Chamorro, 2017), como es el caso de Arica. Dicha influencia se refleja en las formas de relación entre hombres y mujeres y en la expresión de sus simbolismos en carnavales y festividades. Todo lo cual confluye en el espacio público que vivencian comunidades educativas y demás integrantes de la ciudad, incidiendo directamente en la socialización de las nuevas generaciones.

De esta forma podemos apreciar que en las familias aymara existen formas de pensar la vida sexual entre mujeres y hombres que se distancian del modelo hegemónico que la que estandariza (Carrasco et al., 2021), predomina la creencia de que existe igualdad social entre el hombre y la mujer bajo la concepción de que el prestigio tiene un carácter situacional y transitorio. Por tanto, lo femenino y la mujer puede también situarse en rangos superiores respecto de seres masculinos (Carrasco y Gavilán, 2014), no existiendo una relación jerárquica entre ambos.

En este sentido la sexualidad es pensada como una dimensión de los cuerpos humanos que los reproduce biológica, social y simbólicamente formando parte de su sistema de creencias y cosmovisión, donde se reconoce la presencia de una sexualidad no reproductiva como la homosexual, a pesar de su exclusión de la vida "normal" de las personas. Incluso en varias de sus deidades se reconocen tendencias bisexuales o andróginas (Carrasco y Gavilán, 2009). A modo ilustrativo el término $q^{\prime} i w a$ se usa para referirse a los hombres cuando se visten con ropa de mujer para ciertos rituales, representa la conjunción de hombre y mujer, donde los sexos opuestos se mezclan por igual, al mismo tiempo que simboliza la creación de desequilibrio y la ruptura del orden binario (Stobart, 2010).

Estas deidades son representadas en el carnaval Inti Ch'amampi, Con La Fuerza del Sol que desde la década de los ochenta es parte importante de la cultura de Arica y sus 
habitantes. A decir de Chamorro (2017), siguiendo a Canepa (2001) y Citro (2009) esta performance permite comprender estas prácticas expresivas como recursos culturales de comunicación social y simbólica, a través de la cuales los actores andinos buscan autorrepresentarse en un espacio público local y transfronterizo, respondiendo a las imágenes impuestas, con fines contrahegemónicos o bien como mecanismos de autoconstitución o transformación.

Según lo anterior, se puede apreciar que las condiciones de cómo se concibe el género siempre han de considerarse con relación al contexto especifico en que ocurren (Carrasco y Gavilán, 2014) así como los significados que se otorguen a la sexualidad dependen de características socioculturales (Carrasco et al., 2021). De esta forma simbologías, rituales y creencias que están presentes en la cultura ariqueña y por ende en la cultura escolar se intersectan actuando como propiciadores de significaciones que otorgan reconocimiento a las diversidades sexo genéricas. Niños, niñas y adolescentes conviven sin extrañeza con pueblos originarios, migrantes, afrodescendientes, peruanos y bolivianos, convirtiéndose la diversidad sexo genérica en otra imagen más de este caleidoscopio multicultural.

Esta explicación colabora en comprender por qué durante el desarrollo de esta investigación en las cuatro comunidades estudiadas, no se reportaron testimonios de homolesbobitransfobia en la convivencia entre las y los estudiantes, siendo además Arica una zona que registra menos casos de denuncia, si se la compara con otras zonas del país. Como es el caso de la región de Valparaíso, ubicada en la zona centro, que ocupa al nivel nacional de acuerdo con el MOVILH, el primer lugar nacional por más casos de discriminación homo-lesbo-bi-transfóbica con un 40,1 1\% en 2018; 56,1\% en 2019 y 34,8\% en 2020 .

Entonces las características culturales y territoriales de cada comunidad educativa son un aspecto fundamental de profundizar en siguientes investigaciones y de considerar en el diseño de políticas inclusivas que efectivamente fracturen el disciplinamiento heteronormativo imperante en los contextos escolares. Especialmente cuando aún en Chile las políticas públicas al respecto son en general reactivas, tardías, insuficientes e incompletas. De allí que debiesen construirse desde la pertinencia cultural de cada contexto y territorio. A decir de Fernández Murillo (2019) hace falta construir a partir de la deconstrucción, desafiando al mundo binario en que vivimos para romper con las normas de las identidades sexuales que actualmente nos rigen.

Finalmente, como proyecciones de futuras investigaciones resulta necesario ahondar en el conocimiento de cuáles serán los significados respecto de diversidad sexual y de género en las comunidades educativas, luego de que efectuado este estudio entrara en vigor en Chile la Ley de Identidad de Género $\mathrm{N}^{\circ} 21.120$ en diciembre de 2019 que garantiza la no discriminación y regula el cambio de nombre y sexo legal para personas mayores de 14 años. Como también interesa conocer cómo se ha vivenciado la inclusión de las diversidades en contextos de confinamiento y teleeducación debido a la crisis sociosanitaria producto de la pandemia del Covid-19 en 2020 y 2021, y cómo se abordará luego que en junio de 2021 el Ministerio de Salud publicó las recomendaciones para la implementación del programa de acompañamiento para niños, niñas y adolescentes trans y género no conforme. Lo que sin duda también impactará las instituciones educativas y sitúa en otro escenario las demandas por derechos de reconocimiento y no discriminación de quienes expresan identidades más allá de la matriz heterosexual. 


\section{Agradecimientos}

Se agradece a la Vicerrectoría Académica, de Investigación y Postgrado de la Universidad a la que pertenece la Investigadora Responsable, Dra. Verónica Rubio Aguilar, por el apoyo para la realización de este estudio, proyecto categoría regular, código 039942, período 2019-2020.

Se agradece a las personas que voluntariamente participaron de este estudio, contribuyendo al conocimiento de la temática de estudio.

\section{Referencias}

Ahumada, M. Wozny, M. Del Carmen, S. y Zúñiga, M. (2018). Narrativas de construcción identitaria de mujeres trans. Millcayac. Revista Digital de Ciencias Sociales, 5(9), 227-256.

Alcívar, N. y Cedeño M. (2018). La diversidad de género en el sistema de educación superior: Causa y efecto. Revista Atlante. Cuadernos de Educación y Desarrollo.

Bard Wigdor, G. y Magallanes, M. L. (2018). El masculinismo hetero-hegemónico argentino y su estrategia desde el ciberactivismo. Masculinities and Social Change, 7(1), 25-51. https://doi.org/10.17583/mcs.2018.2827

Barrientos, J. y Echagüe, C. (2018). El baile de las que sobran. Interrogando la violencia homofóbica en un liceo público chileno. Forum. Qualitative Social Research, 19(1), 1-44. https://doi.org/10.17169/fqs-19.1.2546

Blanco, R. (2006). La equidad y la inclusión social: Uno de los desafíos de la escuela de hoy. REICE. Revista Iberoamericana sobre Calidad, Eficiencia y Cambio en Educación, 4(3), 7-34.

Bruner, J. (2006). Actos de significado: Más allá de la revolución cognitiva. Alianza.

Butler, J. (2002). Cuerpos que importan. Paidós.

Butler, J. (2007). El género en disputa. Paidós.

Canales, M. (2006). Metodologías de investigación social. Introducción a los oficios. Ediciones Lom.

Carrasco, A. y Gavilán, V. (2009). Representaciones del cuerpo, sexo y género entre los aymara del norte de Chile. Chungará, 4l(1), 83-100.

Carrasco, A. y Gavilán, V. (2014). Género y etnicidad: Ser hombre y ser mujer entre los aymara del altiplano chileno. Diálogo Andino. Revista de Historia, Geografía y Cultura Andina, 45, 169180. https://doi.org/10.4067/s0719-26812014000300014

Carrasco, A., Gavilán, V., Vigueras, P. y Vásquez, M. (2021). Significados de las prácticas sexuales entre aymara chilenos. Aportes para reflexionar sobre los cuidados transculturales. Revista Antropologías del Sur, 8 (15), 23-32. https://doi.org/10.25074/rantros.v8i15.1941

Comisión Internacional de Juristas. (2007). Principios de Yogyakarta sobre la aplicación de la legislación internacional de derechos humanos en relación con la orientación sexual y la identidad de género. https://www.refworld.org.es/docid/48244e9f2.html

Cornejo, J. (2014). Bullyng homofóbico en Chile: Trayectoria histórica. Límite. Revista Interdisciplinaria de Filosofía y Psicología, 30(9), 61-70.

Cornejo, J. (2017). Disidencias sexuales en el sistema escolar chileno: represión e invisibilización. Educação e Pesquisa, 43(3), 879-898. https://doi.org/10.1590/s1517-9702201707166973 
Cornejo, J. (2018). Discriminación y violencia homofóbica en el sistema escolar: Estrategias de prevención, manejo y combate. Revista Brasileira de Educação, 23, 1-24.

https://doi.org/10.1590/s1413-24782018230031

Consejo Nacional para Prevenir la Discriminación. (2016) Glosario de la diversidad sexual, de género y características sexuales. CONAPRED.

Chamorro, A. (2017). Imagen y experiencia: El carnaval de Arica como autorrepresentación festiva. Chungara. Revista de Antropología Chilena, 49(1), 121-132. https://doi.org/10.4067/SO717-73562017005000002

Chávez, M., Zapata, J., Petrzelová, J. y Villanueva, G. (2018). La diversidad sexual y sus representaciones en la juventud. Psicogente, 21(39), 62-74. https://doi.org/10.17081/psico.21.39.2822

Del Pino Rubio, S. y Verbal Stockmeyer, V. (2015). La identidad de género en Chile, problematizando el debate. Liminales, 1 (7), 156-178.

Dinis, N. (2011). Homofobia e educação: quando a omissão também é signo de violencia. Educar em Revista, 3, 39-50. https://doi.org/10.1590/S0104-40602011000100004

Espinar, E. (2009). Infancia y socialización estereotipos de género. Padres y Maestros, 326, 17-21.

Espinoza, R. (2016). La visión de los futuros profesores y profesores formadores acerca de asuntos LGBTI en la formación docente. [Trabajo Fin de Máster]. Universidad Central.

Espinoza, M. y Rodríguez, J. (2020) Estudiantes LGBT+ y profesores universitarios. Prácticas de inclusión y exclusión en la educación superior. Voces y Silencios: Revista Latinoamericana de Educación, 11(2), 7-29. https://doi.org/10.18175/VyS11.2.2020.1

Fernández Murillo, M. S. (2019). Reflexiones jaladas de los pelos: Retóricas en torno a la estética del cabello. En M. S. Fernández y V. Oros (Eds.), Estéticas. Pieles, objetos y cuerpos (pp. 96110). MUSEF Editores.

Flick, U. (2012). Introducción a la investigación cualitativa. Morata.

Flores, R. (2009). Observando observadores: Una introducción a las técnicas cualitativas de investigación social. Ediciones Universidad Católica de Chile.

Fundación Todo Mejora. (2016). Encuesta nacional de clima escolar en Chile 2016. Fundación Todo Mejora.

Fundación Todo Mejora. (2017). Enseñando diversidad: Manual de apoyo a profesores, tutores y apoderadxs para enseñar sobre diversidad, orientación sexual e identidad y expresión de género a niñxs y adolescentes entre 9 y 12 años. Fundación Todo Mejora.

Fundación Todo Mejora. (2019). La calidad de vida y bienestar de estudiantes LGBTIQ+. Fundación Todo Mejora.

Galaz, C., Sepúlveda, M, Poblete, R., Troncoso, L., Morrison, R. (2018). Derechos LGTBI en Chile: Tensiones en la constitución de otredades sexualizadas. Psicoperspectivas, 17(1), 65-81. https://doi.org/10.5027/psicoperspectivas-vol17-issue 1-fulltext-1 165

García-Orriols, J. y Torrebadella-Flix, X. (2019). Homofobia y orientación sexual en deportistas federados en cataluña: inmersos en el legado de la heteronormatividad. masculinities and social. Change,8(3), 222-250. https://doi.org/10.17583/mcs.2019.4308

Gergen, K. (2006). Realidades y relaciones: Aproximación a la construcción social. Paidós.

Hill Collins, P. (2017). La diferencia que hace el poder: Interseccionalidad y democracia participativa. Revista de Investigaciones Feministas, 8(1), 19-39.

https://doi.org/10.5209/INFE.54888 
Hill Collins, P. y Bilge, S. (2016). Interseccionalidad. Morata.

Honneth, A. (2009). Crítica del agravio moral: Patologías de la sociedad contemporánea. Universidad Autónoma Metropolitana. https://doi.org/10.2307/j.ctvm7bcdz

Honneth, A., Rancière, J. y Genel, K. (2016). Recognition or disagreement: A critical encounter on the politics offreedom, equality, and identity. Columbia University Press. https://doi.org/10.7312/honn 17716

Julio, C., Kaeuffer, A., Riquelme, C., Silva, M., Osorio, M. y y Torres, E. (2016). Conocimientos sobre identidad sexual de profesores y profesoras. ¿Barreras o facilitadores de construcción identitaria? Revista Latinoamericana de Educación Inclusiva, 10(2), 53-71. https://doi.org/10.4067/S0718-73782016000200005

Lagares, P. y Puerto, J. (2001). Población y muestra. Universidad de Sevilla.

Laviosa, V. (2016). Realización de un cortometraje que retrate a la generación " $\Upsilon$ ” (millennials) usando la estética hipster. [Trabajo Fin de Grado]. Universidad Católica Andrés Bello.

Martínez, C. Tomicic, A., Gálvez, C., Rodríguez, J., Rosenbaum, C. y Aguayo, F. (2018). Psicoterapia culturalmente competente para el trabajo con pacientes LGBT+. Una guía para psicoterapeutas y profesionales de la salud mental. CEPPS-UDP.

Ministerio de Educación. (2017). Orientaciones para promover espacios de participación y sana Convivencia Escolar. MINEDUC.

Ministerio de Educación. (2017). Orientaciones para la inclusión de las personas lesbianas, gays, bisexuales, trans e intersex en el sistema educativo chileno. MINEDUC.

Ministerio de Salud. (2021). Recomendaciones para la implementación del programa de acompañamiento para niños, niñas y adolescentes trans y género no conforme. MINSAL.

Movimiento de Liberación e Integración Homosexual. (2010). Educando en la Diversidad Orientación sexual e identidad de género en las aulas. MOVILH.

Movimiento de Liberación e Integración Homosexual. (2018). XVII Informe anual de derechos humanos, diversidad sexual y de género en Chile. MOVILH.

Movimiento de Liberación e Integración Homosexual. (2019). XVIII Informe anual de Derechos humanos, diversidad sexual y de género en Chile. MOVILH.

Movimiento de Liberación e Integración Homosexual. (2020). XIX Informe anual de derechos humanos, diversidad sexual y de género en Chile. MOVILH.

OTD. (2017). Encuesta T. OTD.

OTD. (2017). Ni hombres, ni mujeres: Existir más allá del binario de género. OTD.

Preciado, B. (2002). Manifiesto contra-sexual. Opera Prima.

Ramírez, M. y Contreras, S. (2016). Narrativas de identidad afectivo-sexual LGBTIQ+ en contextos escolares: El aparecer frente al otro. Estudios Pedagógicos, 42(1) 235-254. https://doi.org/10.4067/So718-07052016000100015

Ravetllat, I. (2018). Igual de diferentes: La identidad de género de niñas, niños y adolescentes en Chile. Ius et Praxis, 24(1), 397-436. https://doi.org/10.4067/S07 18-00122018000100397

Revuelta, B. y Hernández, R. (2019). La teoría de Axel Honneth sobre justicia social, reconocimiento y experiencias del sujeto en las sociedades contemporáneas. Cinta Moebio, 66, 333-346. https://doi.org/10.4067/S0717-554X2019000300333 
Rivera, M. (2016). Interseccionalidad e inclusión en la educación superior: Consideraciones sobre la Universidad Nacional de Colombia. Pedagogía y Saberes, 44, 105-118. https://doi.org/10.17227/01212494.44pys 105.118

Rojas, M. T., Fernández, M. B., Astudillo, P., Stefoni C., Salinas P. y Valdebenito M. J. (2019). La inclusión de estudiantes LGTBI en las escuelas chilenas. Entre invisibilización y reconocimiento social. Pensamiento Educativo. Revista de Investigación Educacional Latinoamericana, 56(1), 1-14. https://doi.10.7764/PEL.56.1.2019.3

Romero, C. y Montenegro, M. (2018). Políticas públicas para la gestión de la diversidad sexual y de género: Un análisis interseccional. Psicoperspectivas, 17(1), 11-34. https://doi.org/10.5027/psicoperspectivas-vol17-issue1-fulltext-1211

Rubio, V., Basáez, F., Escorza, E. y Fuenzalida, G. (2021). Identidad de género de jóvenes trans: Perpetuación y pérdida de privilegios patriarcales. Masculinities and Social Change, 10(3), 242-269. https://doi.org/10.17583/mcs.7375

Salas, N. y Salas M. (2016). Tiza de colores: Hacia la enseñanza de la inclusión sobre diversidad sexual en la formación inicial docente. Revista Latinoamericana de Educación Inclusiva, 1O(2), 73-91. https://doi.org/10.4067/S07 18-73782016000200006

Salgado, A. (2007). Investigación cualitativa: Diseños, evaluación del rigor metodológico y retos. Liberabit, 13, 71-78.

Sánchez González, S. y González-de-Garay, B. (2020). La diversidad afectivo-sexual en first dates. Análisis de contenido del perfil de los participantes. Masculinities and Social Change 9(1), 113147. https://doi.org/10.17583/mcs.2020.3629

Scribano, A. (2008). El proceso de investigación social cualitativo. Prometeo.

Stake, R. E. (2005). Qualitative case studies. En N. Denzin e Y. Lincoln (Eds.), The SAGE handbook of qualitative research (pp. 443-466). SAGE.

Stobart, H. (2010). Tara y Q'iwa: Mundos de Sonidos y Significados. En A. Gerard (Ed.), Diablos tentadores y pinkillus embriagadores en la fiesta de Anata Phujllay (pp. 25-40). Editores Plurales.

Strauss, A. y Corbin, J. (2002). Bases de la investigación cualitativa: Técnicas y procedimientos para desarrollar la teoría fundamentada. Universidad de Antioquia.

Superintendencia de Educación. (2017). Circular $N^{\circ} 0768$ de 2017 que establece derechos de niñas, niños y estudiantes trans en el ámbito de la educación. https://www.supereduc.cl

Superintendencia de Educación. (2018). Denuncias por materia específica, nivel educacional del afectado y trimestre. https://www.supereduc.cl

Toro, E., Moya, P. y Poblete, R. (2020). Representaciones sociales de profesores y estudiantes sobre las personas LGBTI: Un estudio de caso en un liceo católico y laico. Revista Latinoamericana de Educación Inclusiva, 14(1), 37-51 https://doi.org/10.4067/S0718-73782020000100037

Trujillo, A. (2014). Escuela para la diversidad: Investigación sobre experiencias y efectos de las políticas de inclusión LGBT en escuelas secundarias. Universidad Nacional de la Plata.

UNESCO. (2005). Guiderlines for inclusion: Ensuring Acces to education for All. UNESCO.

UNESCO. (2012). Educación Integral de la Sexualidad: Conceptos, Enfoques y Competencias. UNESCO.

UNESCO. (2016). Abiertamente: Respuestas del sector educación a la violencia basada en la orientación sexual e identidad/ expresión de género. UNESCO.

UNESCO. (2017). Abiertamente: Respuestas del sector educación a la violencia basada en la orientación sexual e identidad/ expresión de género. UNESCO. 
Vasilachis, I., Ameigeiras, A., Chernobilsky, L., Giménez V., Mallimaci, F., Mendizábal, N., Neiman, G. y Sonería, A. (2006). Estrategias de investigación cualitativa. Editorial Gedisa.

Vidal Ortiz, S. (2014). Corporalidades trans: Algunas representaciones de placer y violencia en AL. Interdisciplina, 2(3), 26-42. https://doi.org/10.22201/ceiich.24485705e.2014.3.47789

Vidal Velis, F., Pérez, I., Barrientos, J. y Gutiérrez, G. (2020). Educación en tiempos del género. Consideraciones en torno a una educación no sexista y no generista. Revista Latinoamericana de Educación Inclusiva, 14(2), 21-37. https://doi.org/10.4067/s0718-73782020000200021

Viveros, M. (2016). La interseccionalidad: Una aproximación situada a la dominación. Debate Feminista, 52, 1-17. https://doi.org/10.1016/j.df.2016.09.005

\section{Breve CV de las autoras}

\section{Verónica Rubio Aguilar}

Asistente Social y Licenciada en Servicio Social, Universidad de Valparaíso, Magister en Educación y Doctora en Educación, Universidad de Playa Ancha de Ciencias de la Educación, Valparaíso, Chile. Directora Carrera de Trabajo Social, Universidad Santo Tomás, Viña del Mar e Investigadora asociada del Centro CIELO de Investigación en Familia, Trabajo y Ciudadanía, Facultad de Ciencias Sociales y Comunicaciones, Universidad Santo Tomás. Académica de pre y postgrado, investigadora principal y autora de varias publicaciones científicas en Responsabilidad Social, Inclusión, Educación y Trabajo Social. Email:vrubioa@santotomas.cl

ORCID ID: https://orcid.org/0000-0002-8965-1461

\section{Patricia Miranda Mamani}

Trabajadora Social, Licenciada en Trabajo Social, Magister en Intervenciones y Estudios Avanzados en Trabajo Social, Universidad Santo Tomás. Académica carrera de Servicio Social del Instituto Profesional Santo Tomás, Arica. Se ha desempeñado laboralmente en los ámbitos de familia, infancia, adolescencia y educación. Actualmente trabaja en la Secretaría Regional Ministerial de Salud Arica y Parinacota, Unidad de Epidemiología de Campo. Testeo, Trazabilidad y Aislamiento-TTA COVID-19. Email: pmiranda10@santotomas.cl

ORCID ID: https://orcid.org/0000-0002-1236-6590

\section{Genoveva Tiayna Pacha}

Trabajadora Social, Licenciada en Trabajo Social, Magister en Educación, mención Gestión Inclusiva, Magister en Intervenciones y Estudios Avanzados en Trabajo Social, Universidad Santo Tomás. Académica carrera de Servicio Social del Instituto Profesional Santo Tomás, Arica. Se ha desempeñado laboralmente como trabajadora social en el ámbito de educación y como docente en carreras técnicas y profesionales a cargo de asignaturas teóricas y prácticas. Actualmente es Coordinadora de la Clínica Socio Jurídica de Santo Tomás, Arica. Email: genovevatiaynapa@santotomas.cl

ORCID ID: https://orcid.org/0000-0002-6784-7615

\section{Elizabeth Hidalgo Figueroa}


Trabajadora Social, Licenciada en Trabajo Social, Magister en Intervenciones y Estudios Avanzados en Trabajo Social, Universidad Santo Tomás. Académica carrera de Servicio Social del Instituto Profesional Santo Tomás, Arica. Se ha desempeñado laboralmente en los ámbitos de familia, infancia, adolescencia y educación. Actualmente es trabajadora social en establecimientos de educación básica de carácter municipal de la ciudad de Arica. Email: elizabethhidalgofi@santotomas.cl

ORCID ID: https://orcid.org/0000-0001-7646-3552

\section{Carol Tuna Varas}

Trabajadora Social, Licenciada en Trabajo Social, Magister en Intervenciones y Estudios Avanzados en Trabajo Social, Universidad Santo Tomás. Académica carrera de Servicio Social del Instituto Profesional Santo Tomás, Arica. Se ha desempeñado laboralmente como trabajadora social en el ámbito de educación y como docente en carreras técnicas y profesionales a cargo de asignaturas teóricas y prácticas. Actualmente trabaja en el Departamento de Educación Municipal de Arica y es Jefa de carrera del área de Ciencias Sociales del Instituto Profesional Santo Tomás de Arica. Email: caroltunava@santotomas.cl

ORCID ID: https://orcid.org/0000-0003-1303-2872 